\title{
Video Article \\ A Guide to Modern Quantitative Fluorescent Western Blotting with Troubleshooting Strategies
}

\author{
Samantha L. Eaton ${ }^{1}$, Maica Llavero Hurtado ${ }^{1}$, Karla J. Oldknow ${ }^{2}$, Laura C. Graham ${ }^{1}$, Thomas W. Marchant ${ }^{1}$, Thomas H. Gillingwater ${ }^{3,4}$, \\ Colin Farquharson ${ }^{2}$, Thomas M. Wishart ${ }^{1,4}$ \\ ${ }^{1}$ Division of Neurobiology, The Roslin Institute and Royal (Dick) School of Veterinary Studies, University of Edinburgh \\ ${ }^{2}$ Division of Developmental Biology, The Roslin Institute and Royal (Dick) School of Veterinary Studies, University of Edinburgh \\ ${ }^{3}$ Centre for Integrative Physiology, University of Edinburgh \\ ${ }^{4}$ Euan MacDonald Centre for Motor Neurone Disease Research, University of Edinburgh
}

Correspondence to: Thomas M. Wishart at t.m.wishart@ed.ac.uk

URL: https://www.jove.com/video/52099

DOI: doi:10.3791/52099

Keywords: Basic Protocols, Issue 93, western blotting, fluorescent, LI-COR, protein, quantitative analysis, loading control

Date Published: $11 / 20 / 2014$

Citation: Eaton, S.L., Hurtado, M.L., Oldknow, K.J., Graham, L.C., Marchant, T.W., Gillingwater, T.H., Farquharson, C., Wishart, T.M. A Guide to Modern Quantitative Fluorescent Western Blotting with Troubleshooting Strategies. J. Vis. Exp. (93), e52099, doi:10.3791/52099 (2014).

\section{Abstract}

The late 1970s saw the first publicly reported use of the western blot, a technique for assessing the presence and relative abundance of specific proteins within complex biological samples. Since then, western blotting methodology has become a common component of the molecular biologists experimental repertoire. A cursory search of PubMed using the term "western blot" suggests that in excess of two hundred and twenty thousand published manuscripts have made use of this technique by the year 2014. Importantly, the last ten years have seen technical imaging advances coupled with the development of sensitive fluorescent labels which have improved sensitivity and yielded even greater ranges of linear detection. The result is a now truly Quantifiable Fluorescence based Western Blot (QFWB) that allows biologists to carry out comparative expression analysis with greater sensitivity and accuracy than ever before. Many "optimized" western blotting methodologies exist and are utilized in different laboratories. These often prove difficult to implement due to the requirement of subtle but undocumented procedural amendments. This protocol provides a comprehensive description of an established and robust QFWB method, complete with troubleshooting strategies.

\section{Video Link}

The video component of this article can be found at https://www.jove.com/video/52099/

\section{Introduction}

Western blotting (WB) is an analytical technique originally developed in the late 1970s to determine the presence or absence of a protein of interest in a complex biological sample, such as a tissue homogenate ${ }^{1}$. Commonly referred to as the protein immunoblot, due to the key antibody-antigen interaction, the methodology consists of 5 distinct steps: 1) electrophoretic separation of the proteins by their isoelectric point; 2) transfer to a nitrocellulose or polyvinylidene difluoride (PVDF) membrane; 3) labeling using a primary antibody specific to the protein of interest; 4) incubation with a secondary antibody directed against the primary antibody; and 5) visualization.

Visualization methodology has evolved with time to improve safety and sensitivity. Some of the first WBs were carried out using radio labeled tags which then progressed to colorimetric and then the more widely used chemilluminescent (ECL) methods. Radioactivity was directly labeled onto probes for specific antigens whereas colorimetric and ECL methodologies use an indirect labeling technique with an enzyme reporter such as alkaline phosphatase or streptavidin horseradish peroxidase (HRP) ${ }^{2}$. The intensity of labeling of the chromagen or luminescent product is measured using densitometry whereby the signal strength, strong or weak, indicates more or less the presence of the protein of interest in the sample. ECL is the more sensitive and therefore favored methodology ${ }^{2}$ but all 3 methods were initially developed on $x$-ray film with more sophisticated digital imaging techniques subsequently established ${ }^{3}$. The advancement of digital imaging of WB not only allowed a researcher to determine the presence or absence of their protein of interest, but also allowed an inference to the level of expression of a selected protein when compared against other samples and therefore can be referred to as "semi-quantitative". Recently, a truly quantitative and more sensitive western blotting technology has been developed whereby the level of fluorescence measured is directly related to the quantity and expression of a single protein within a sample: quantitative fluorescent western blotting (QFWB).

When comparing QFWB with ECL labeling, the use of a fluorescent secondary antibody generates a linear detection profile ${ }^{4}$. This is in contrast to $E C L$ techniques where signal linearity generally occurs with low protein loads below $5 \mu \mathrm{g}$ and signal saturation directly related to protein expression, i.e., with ubiquitously expressed housekeeping genes ${ }^{5,6}$. This disparity is most likely caused by a greater number of binding sites available for an avidin ECL substrate to bind to a biotinylated secondary, resulting in a higher likelihood of potential signal saturation. This is one of the main reasons for ECL base immunoblotting being referred to as only "semi-quantitative" 7 . The saturation point of signal is of critical importance when measuring subtle differences in expression levels and can lead to inaccurate measurements. In recent years the advent of 
widespread proteomic techniques detailing ever increasing sensitivity and identification of subtle expression differentials has resulted in an ever increasing reliance on truly quantitative western blotting for validation experiments ${ }^{8,9}$. The application of sensitive, robust and truly quantitative methodology is therefore crucial.

Many "optimized" western blotting methodologies have been utilized by independent laboratories, which frequently prove difficult to set up or replicate due to subtle methodological adjustments that may not be apparent in formal documented protocols. This is an established and robust protocol for QFWB and additionally provides valuable strategies for troubleshooting common problems that may arise during implementation.

This protocol was originally optimized for use with murine brain homogenates, but has since been used effectively across a broad range of tissue samples and species ${ }^{4,9,10}$. Potential protocol variations required for specific troubleshooting issues are included.

\section{Protocol}

This protocol has been optimized using commercially produced buffers, gels and transfer stacks in order to reduce variability and improve consistency. Refer to Materials List for a complete list of consumables required.

\section{Fluorescent WB protocol using I-Blot fast transfer and LI-COR Odyssey imaging system}

\section{Preparation of Sample}

1. Buffer selection/preparation

1. Select an appropriate extraction buffer for sample homogenization and ensure that the buffer is compatible with all downstream techniques to be employed. Prepare an extraction buffer: RIPA buffer $(25 \mathrm{mM}$ Tris- $\mathrm{HCl}(\mathrm{pH} 7.6), 150 \mathrm{mM} \mathrm{NaCl}, 1 \% \mathrm{NP}-40,1 \%$ sodium deoxycholate, $0.1 \%$ SDS) containing $5 \%$ protease inhibitor cocktail prior to sample isolation.

NOTE: There are many different types of extraction buffers available and are selected depending upon the location of the protein of interest within the cell. These include but are not limited to RIPA buffer (whole cell, mitochondrial and nuclear components), NP-40 Iysis buffer (whole cell or membrane bound) and Tris-Triton (cytoplasmic skeletal bound). However, some chemical detergents within extraction buffers may help to solubilize or even deactivate a protein but can interfere with protein determination when using a specific protein determination assay, i.e., Bicinchoninic acid (BCA) assay. Check the manufacturer's guidelines regarding chemical compatibility with the assay.

2. Protein extraction/solubilization

1. Manually macerate the tissue sample using scissors and/or scalpel followed by homogenization using either a dounce or a hand-held electric homogenizer with a polypropylene tip in the prepared extraction buffer at approximately 1:10 w/v (tissue weight/ buffer volume) until a smooth/consistent homogenate is produced.

NOTE: For smaller, precious/difficult to obtain samples, detergent based extractions can still be effective down to 1:5.

2. Post homogenization, centrifuge samples at $20,000 \mathrm{xg}$ for $20 \mathrm{~min}$ at $4{ }^{\circ} \mathrm{C}$. Remove the supernatant containing the solubilized proteins and store at $-80^{\circ} \mathrm{C}$ until required. Retain insoluble pellets to allow further more stringent extraction if required later. Proceed to protein determination step 1.3 .

3. Protein determination

1. Determine the concentration of protein within each extracted sample by using either a BCA, Bradford or similar assay. When calculating the standard curve, ensure that the coefficient of determination ratio, R-squared value, is greater than or equal to 0.99 which reflects the most accurate determination of protein abundance within the samples ${ }^{15}$.

NOTE: All samples being compared by QFWB must be assayed against the same standard curve.

4. Preparation of sample

1. Plan and record the loading order of ladders and samples for 2 identical gels: gel 1 for transfer and gel 2 as a loading control. Load the control and "treated" samples sequentially to avoid any bias that could arise with poor or unequivocal transfer.

2. Calculate the protein volume required for each sample. A standard protein load to detect proteins in neuronal isolates is $15 \mu \mathrm{g}$. Make each sample up to a $10 \mu \mathrm{l}$ volume with $\mathrm{dH}_{2} \mathrm{O}$. Add $5 \mu \mathrm{l}$ of loading buffer, vortex and heat at $98^{\circ} \mathrm{C}$ for $2 \mathrm{~min}$.

3. Include a positive control sample such as a recombinant protein to aid with identification of the correct band of interest. However prepare the positive control sample correctly following the manufacturer's guidelines to avoid selection of the wrong bands. See Figure 1. 

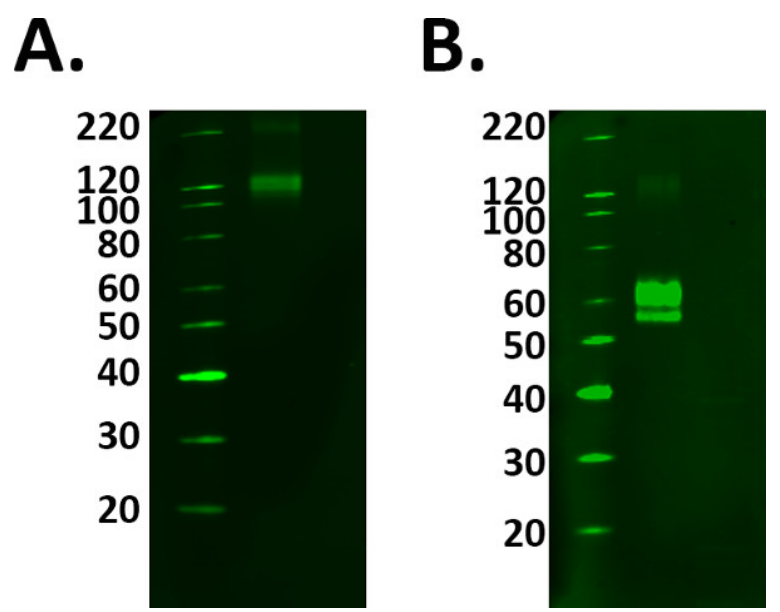

Figure 1. Positive control selection. The addition of positive controls to an experiment confirms the labeling detected is real. However, caution must be taken to ensure your control is working correctly prior to using experimental samples. A) Fusion protein TREM 2 was loaded at the manufacturer's guidelines of $1 \mu \mathrm{g} / \mathrm{ml}$, however the labeling observed at $110 \mathrm{kDa}$ conflicts with the datasheet predicted molecular weight of 60-70 kDa. B) After addition and incubation with the reducing agent, the fusion protein labeling was detected at the predicted molecular weight. However, this meant a greater protein load was required as the reduction process decreased the signal of the protein.

\section{Electrophoretic Separation of Proteins}

1. Preparation of $4-12 \%$ Bis/tris gel $(1.0 \mathrm{~mm})$

NOTE: Gradient gels are used to produce greater protein separation across a broad molecular weight range.

1. Select and prepare MES or MOPS running buffer $\left(1 \mathrm{~L}\right.$ per tank) by diluting with $\mathrm{dH}_{2} \mathrm{O}$. MES buffer produces better resolution of proteins within 3.5-160 kDa whereas MOPs running buffer is preferred to detect higher molecular weight proteins above $200 \mathrm{kDa}$ however will have poorer resolution of lower molecular weight proteins below $15 \mathrm{kDa}$.

2. Remove the comb and strip of tape that covers the foot of the gel. Gently wash the wells out with running buffer using a $1 \mathrm{ml}$ pipette. Fill the wells with running buffer and ensure no bubbles remain in the gel.

2. Gel preparation and loading

1. Insert the gels into the tank and fill the inter gel compartment with running buffer to ensure all seals are working effectively.

2. Add $3 \mu \mathrm{l}$ of molecular weight standard into the first well in gel 1 and gel 2 . Load $10 \mu \mathrm{l}$ of each sample into subsequent wells. NOTE: With the exception of the ladders, gel 1 \& gel 2 must be identical. It is imperative that the pre-stained molecular weight standard used for gel 2 is blue and visible when using a total protein stain in order to be visible in the 700 channel of Odyssey imager.

3. Once both gels are loaded, fill the tank with the remaining running buffer and secure the lid.

3. Electrophoresis

1. "Run" the gel at $80 \mathrm{~V}$ for $4 \mathrm{~min}$ to ensure the sample enters the polyacrylamide gel matrix in a uniform fashion. Thereafter increase the voltage and time to $180 \mathrm{~V}$ for 50 min respectively or until the sample dye can be seen at the foot of the gel. NOTE: Higher voltages can be applied to achieve shorter run times. However, this may result in "smiley" gels where the middle samples of the gels run faster than the outer lane samples caused by an increase in temperature ${ }^{14}$.

\section{Total Protein Stain of the Loading Control Gel}

1. Release gel 2 from the cassette using a gel knife. Remove the wells and the foot of the gel. Mark the gel to aid orientation.

2. Decant approximately $30 \mathrm{ml}$ of the protein stain into a square Petri dish (approximate size $12 \times 12 \mathrm{~cm}$ ). Place gel 2 into the protein stain, ensuring the solution covers the entire gel, then agitate the gel for $1 \mathrm{hr}$ minimum at room temperature to stain.

3. Decant the protein stain and wash the gel $3 x$ in distilled water prior to visualization. Proceed to step 6.3 to visualize.

\section{I-Blot Semi Dry Fast Protein Transfer}

NOTE: All reagents required for protein transfer using the I-Blot machine are commercial products specifically designed for the I-Blot methodology and can be found in the Materials List.

1. Prepare the "bottom" transfer stack. Remove foil cover and pre-wet with running buffer direct from the gel tank. Pre-wet filter paper with distilled water.

2. Repeat step 3.1 and place gel 1 onto the prepared "bottom" transfer stack.

3. Lay the pre-wet filter paper over the gel. Roll the filter paper and gel to ensure no bubbles are trapped between the layers.

4. Remove the foil lid from the top transfer stack and lay the top stack on top of the filter paper and bottom stack. Roll the transfer stack "sandwich" to eliminate any air bubbles.

5. Insert the sponge from the transfer stack kit onto the lid of the I-Blot machine. Place the transfer stack sandwich onto the dedicated space on the I-Blot machine then close lid securely. Start the I-Blot and transfer for $8.5 \mathrm{~min}$ on program 3. 
6. If there is a low protein signal or uneven high: low molecular weight protein ratios, test the manufacturer's transfer times when using a semi dry "fast" transfer method. Run a simple optimization protocol using sample repeats of identical load to determine the ideal transfer voltage/ time combination as seen in Figure 2.

A.

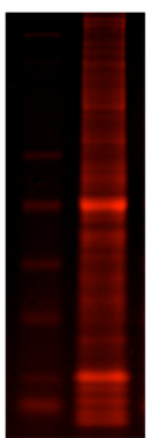

NO

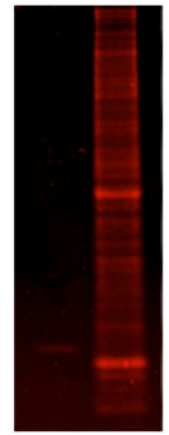

$7.5 \mathrm{MIN}$

TRANS

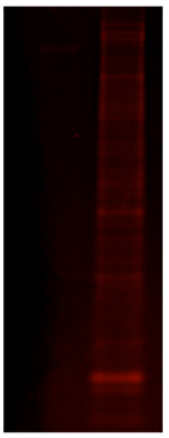

8.5 MIN

TRANS

B.

Figure 2. Optimization of transfer using the I-Blot. A) A single gel loaded with ladder and $15 \mu \mathrm{g}$ of murine whole brain homogenate in three tandem repeats was cut into three sections. One section was not transferred, one was transferred for 7.5 min (as per manufacturer's guidelines) and one for $8.5 \mathrm{~min}$. The gel sections were then stained with Instant Blue protein stain, scanned on an infrared imager in the 680 channel and quantified. B) Graphical representation of the quantification values demonstrating the difference in residual protein content of each gel following $0,7.5$, and $8.5 \mathrm{~min}$ of transfer. Note that an additional minute of transfer time resulted in additional protein transfer of approximately $45 \%$. Please click here to view a larger version of this figure.

\section{Antibody Detection of Proteins}

1. Membrane preparation and blocking

1. Remove transfer stack from the I-Blot machine. Remove the top and filter paper layer exposing the gel on the bottom transfer stack.

2. Cut around the gel with a scalpel and ensure that a triangular cut for orientation purposes is represented on the membrane. Following membrane trimming, protein stain the gel to check transfer efficiency and/or discard.

3. Swiftly move the membrane into a clean $50 \mathrm{ml}$ tube (or equivalent receptacle) containing $5 \mathrm{ml}$ of $1 \times$ PBS and place onto a mechanical roller (or orbital platform) for constant agitation.

NOTE: It is critical that the membrane is not allowed to dry out. During semi-dry transfer the membrane will become hot. Wait 2 min before opening the transfer stack as this will allow it to cool and slow drying time during stack deconstruction. Use of a roller and tube for agitation during washes and incubations allows significant reduction in the volumes of solutions required.

4. Wash the membrane $3 \times 5 \mathrm{~min}$ in $1 \times$ PBS. Discard the 1x PBS and block the membrane with undiluted blocking buffer for a minimum of $30 \mathrm{~min}$ at room temperature.

2. Primary antibody preparation.

1. Prepare the primary antibody according to the manufacturer's guidelines in $5 \mathrm{ml}$ of blocking buffer with $0.1 \%$ Tween 20 . Incubate the membrane with the prepared primary antibody overnight at $4{ }^{\circ} \mathrm{C}$ with constant agitation.

NOTE: Blocking buffer is generally used undiluted but may be diluted up to 1:4 with PBS if the primary antibody has high enough specificity.

2. Discard the primary antibody and wash the membrane 6 times for 5 min each with 1x PBS.

3. Secondary antibody selection \& preparation

1. For secondary only labeling assessment for control purposes, omit steps 5.2 .1 and 5.2 .2 above and proceed to step 5.3 to determine sample specific secondary banding patterns. See Figure 3. 


\section{Goat anti rabbit $\mathbf{8 0 0}$}

A.

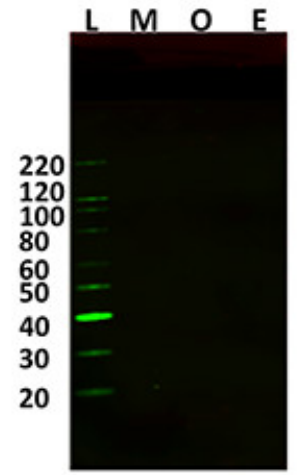

B.

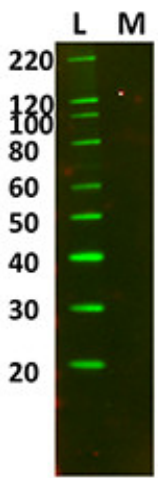

\section{Goat anti} rabbit 680

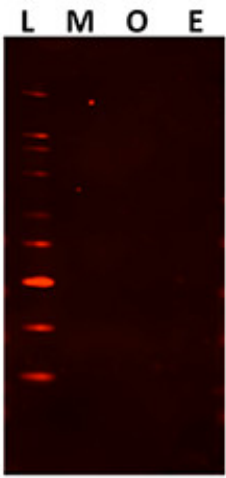

L M

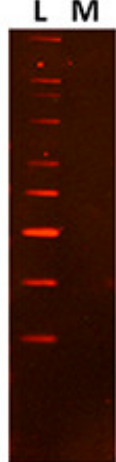

\section{Donkey anti goat 800}

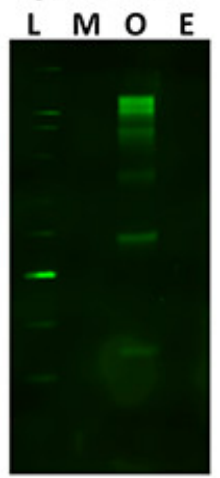

L $M$

\section{Donkey anti mouse 680}

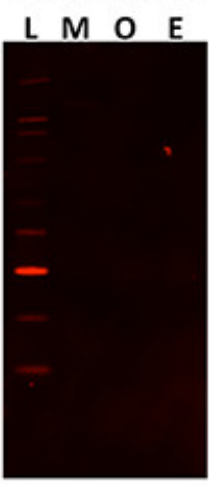

L $M$

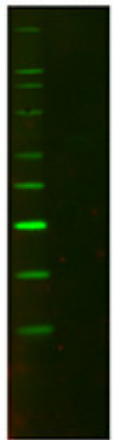

Figure 3. Optimization of secondary antibodies. A) A multi species comparison of secondary only non-specific labeling against $15 \mu \mathrm{g}$ of murine $(M)$, Ovine $(O)$ and Equine $(E)$ nervous tissue homogenates with a variety of fluorescent tagged secondary antibodies. $L$ is the molecular weight ladder. Ovine tissue homogenate was the only sample to cross-react with the secondary labeling when using donkey anti-goat 800 antibody. B) It is also important to ascertain if non-specific labeling occurs when using a different tissue sample, i.e., murine gastrocnemius muscle $(15 \mu \mathrm{g}$ load). This sample cross reacts with the donkey anti-mouse 680 secondary antibody, however this did not occur when using mouse brain homogenate.

2. Prepare the fluorescent tagged secondary antibody 680 or 800 (red or green channel) following manufacturer recommended concentrations in blocking buffer with $0.1 \%$ Tween 20 . Incubate the membrane in the dark with the prepared secondary antibody for 90 min at room temperature with constant agitation.

3. Discard the secondary antibody and wash the membrane 6 times for 5 min per wash with $1 \times$ PBS. Proceed to step 6 - visualization.

1. If visualization of markers is not working as expected, exam ladders with a range of secondary antibodies as a suitable additional step. Not all secondary antibodies allow visualization of all marker bands in all commercially available ladders.

2. If expected bands are not visible, use an alternative secondary host, i.e., donkey anti rabbit versus goat anti rabbit represents a suitable protocol modification. See Figure 4. Host species used to raise a secondary antibody can occasionally cause an issue with visualization due to lack of specificity for specific primary antibodies. 


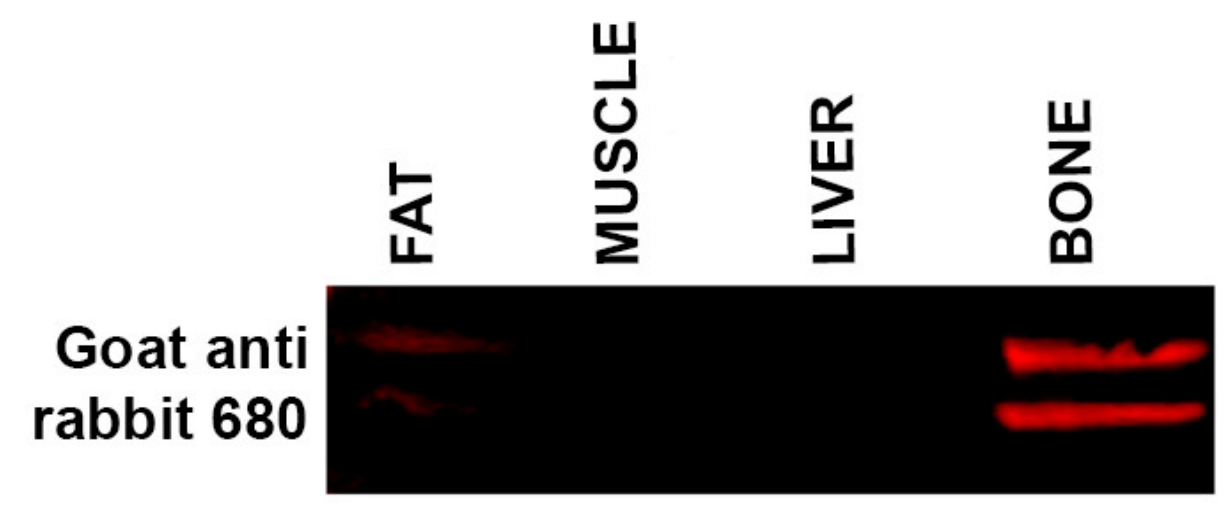

\section{Goat anti rabbit HRP}

\section{Donkey anti rabbit 680}

Figure 4. Troubleshooting secondary antibody specificity. Western blot of a range of tissue samples (15 $\mu$ g protein per lane) - fat, muscle, liver and bone - incubated with ERK primary antibody and incubated with three different secondary antibodies. Top panel: WB labeled with LI-COR goat anti-rabbit 680 secondary antibody produced weak labeling of fat and bone and no signal was detected in the liver and muscle samples. Middle panel: Membrane (from top panel) was stripped and reprobed using ECL methodology and Goat anti-rabbit HRP linked secondary. Bands are now visible in muscle and liver samples and labeling appears more intense in the fat and bone samples. Bottom panel: Membrane (from top and middle panel) was stripped and reprobed using LI-COR Donkey anti-rabbit 680 secondary antibody which has shown a greater affinity for the ERK primary antibody. Labeling for muscle and liver is now visible with increased signal intensity from both fat and bone samples. Please click here to view a larger version of this figure.

\section{Visualization}

NOTE: All images are acquired using the LI-COR Odyssey Classic imager and associated Image Pro analysis software (version 3.1.4).

1. Turn and log on to the computer and imager. Open the imaging software and create a new file. See Figure 5. 


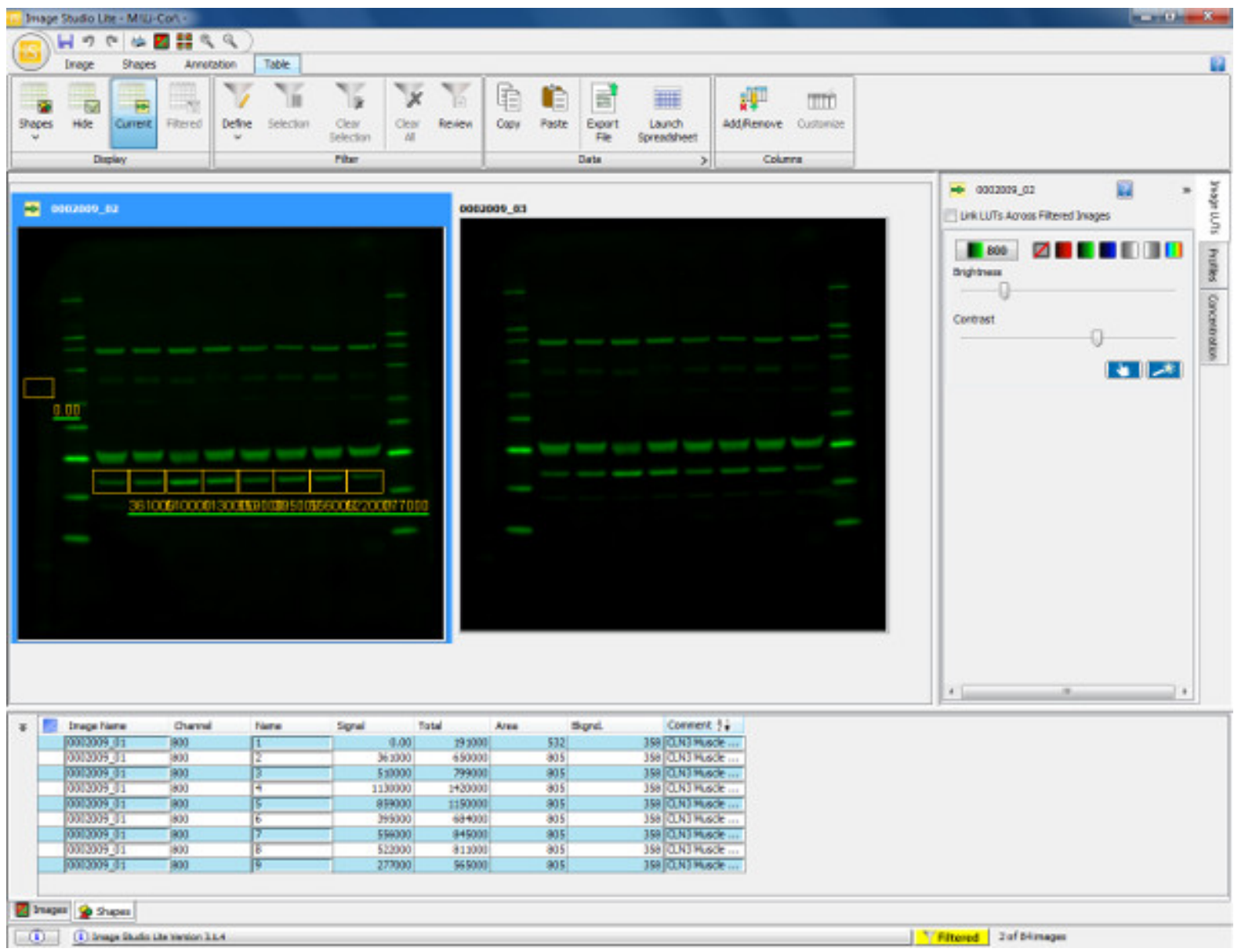

Figure 5. Visualization and quantification of western blot. Scan of a western blot showing murine gastrocnemius muscle ( $30 \mu \mathrm{g}$ load) probed with Annexin $\mathrm{V}$ primary antibody ( $36 \mathrm{kDa}$ ) and goat anti-rabbit 800 secondary antibody. The membrane was scanned and visualized in the 800 channel. To quantify the protein (Annexin V), a rectangular box is drawn around the band of interest from sample 1 . This is then copied and pasted over the remaining sample lanes to ensure measurement of the same area. Background is automatically accounted for around the shape drawn but this can be altered to ensure the background measurement is accurately defined. The table below displays the quantified measurements of each shape drawn including total signal obtained, background and signal with background subtracted. This information can then be exported into a spreadsheet program to calculate expression ratios (as determined by relative fluorescence intensity) and allows subsequent statistical analyses to be performed. Please click here to view a larger version of this figure.

2. Open the lid of the imager. Pour a small amount of $1 \times$ PBS on to the glass on the bottom left hand corner and then place the membrane on top of the PBS. Ensure the membrane is square with the axis on the imager and a $1 \mathrm{~cm}$ gap is left between the membrane and the grid axis.

1. Roll the membrane to ensure no bubbles are trapped between the glass and the membrane.

2. Count the number of squares the membrane occupies on the $x$ and $y$ axis. Close the lid of the imager. Enter the number of squares the membrane occupies on the computer software.

3. Select the appropriate channel to scan the membrane which will depend on the fluorescent tag of the secondary antibody, i.e., 700 channel or 800 channel when a 680 or 800 fluorescent tagged antibody has been used respectively. When dual labeling is carried out, scan in both channels.

NOTE: Optimize single protein labeling prior to carrying out dual labeling.

4. Select the intensity to scan the membrane, if a high abundance protein use a low intensity scan. Alternatively if the primary antibody has poor affinity for the protein of interest select a higher intensity scan, i.e., level 5. Press start to begin the scan. Proceed to step 6.4.

3. Visualization of the loading control gel (Figure 6).

A.

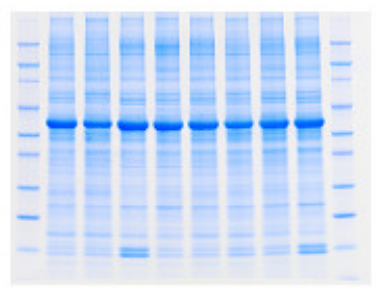

B.

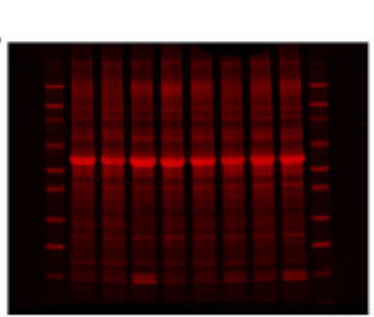

C.

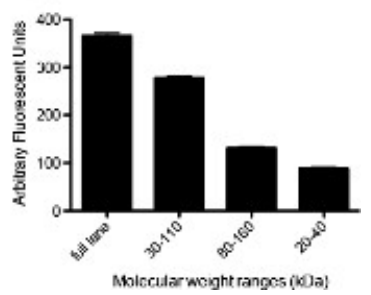


Figure 6. Total protein labeling and analysis. A) Photograph of a total protein labeled gel containing $20 \mu \mathrm{g}$ of equine cervical ganglia homogenate per lane. B) Gel from Panel A scanned in the 680 channel. C) Graph of quantified measurements from the total protein stained gel obtained using imaging software. A range of measurements determined by molecular weight markers, i.e., 30-110 kDa, are taken to provide a histogram of measurements to ensure standard error (SEM) is low (of the grouped samples) indicating that total protein levels in each sample are uniform across the gel. Please click here to view a larger version of this figure.

1. Follow steps 6.1 and 6.2

2. Pour distilled water onto the glass in the bottom corner of the imager where the grid axis begins.

3. Place the gel on top of the water and maneuver so the lanes are perpendicular to either the $x$ or $y$ axis of the grid. Leave a $1 \mathrm{~cm}$ gap between the $y$ and $x$ axis of the grid and the gel and count the number of squares the gel occupies on the grid axis. Close the lid of the imager.

4. Enter the number of squares the gel occupies on the computer software.

5. Select the 700 channel to scan the total protein stained gel. NOTE: Blue fluoresces in the 700 channel.

6. Set the intensity to 5 and press start.

4. Quantification of scanned image

1. Adjust the brightness and contrast buttons to produce the best image quality. If there appears to be high background noise, rescan the membrane at a lower intensity and use the high quality scan setting. NOTE: Any adjustments will not alter the fluorescent data acquired when scanning.

2. Rotate images $90^{\circ}, 180^{\circ}$ and $270^{\circ}$ in order to view in the desired orientation with no alteration to the data acquired. However, when making small adjustments of orientation in "free rotation", by greater than $3^{\circ}$, data acquired in pixilation values can become distorted and thus affect the quantification results.

3. Select a shape from the shapes menu - use a rectangle in most cases - and draw around the band of interest (WB) or the entire lane (total protein gel) in sample lane 1.

4. Copy and paste the shape across to sample lane 2 band of interest or entire lane. Repeat across each individual band of interest for every sample and each lane for total protein gels.

5. Check that the background measurement does not incorporate a signal in the next lane. The background can be automatically deducted by the software and encompass entire edge around the shape drawn or it can be specified to be top/bottom or left and right of the shape. Alternatively, designate a user defined background box.

6. Display the signal for the shapes table for each shape drawn in arbitrary fluorescent units. The background will automatically be deducted from the signal.

7. Export the image as a TIF file to view in different software. Do not export the image and manipulate using non image pro software as this will alter the original data acquired by the imager generating false data.

8. Repeat steps 6.4.3-6.4.7 when quantifying dual labeling or carrying out multiple measurements on loading control gels to generate and collate standard error data.

\section{Post Visualization}

1. Keep membranes in 1x PBS or dry out for long-term storage for future re-probing and stripping of membranes for other proteins of interest.

2. Stripping and re-probing of membranes. See Figure 7.

A.
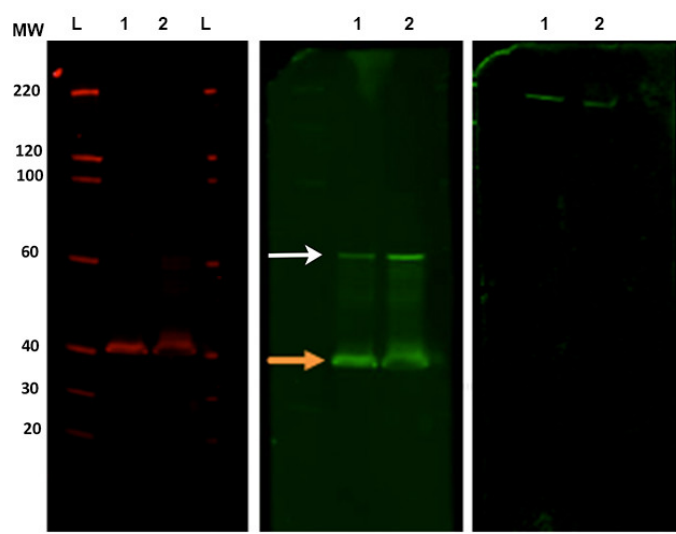

C.

D.

E.

F.

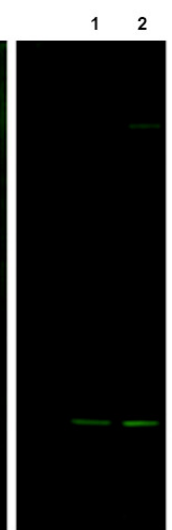

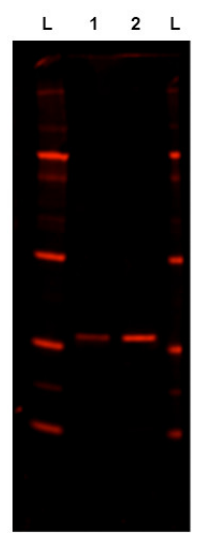

Figure 7. Membrane stripping and reprobing. Representative examples of membranes following various stripping steps scanned with an infrared imaging system. A. The first immunodetection was carried out with CSP primary antibody (rabbit) and scanned at 700 channel. B. First stripping and reprobing with ROCK2 (rabbit) with 800 channel. Orange arrow indicates remaining CSP primary antibody present after the strip and re-probe. This does not affect the measurement of ROCK2 as the band is present at a higher molecular weight (white arrow). C. Second stripping and reprobed with $\beta$-Spectrin antibody (goat) and visualized in the 800 channel. D. Third stripping and reprobing with $\alpha$ synuclein antibody (rabbit). E. Fourth stripping and reprobing with ubiquitin antibody (mouse) with 800 channel. There is still remaining signal from the last antibody ( $\alpha$-synuclein. Orange arrow). F. Fifth stripping and reprobing with CALB2 antibody (rabbit) imaged in $700 \mathrm{channel}$. Secondary antibodies used were: goat anti-mouse $800 \mathrm{cw}$, goat anti-rabbit $680 \mathrm{rd}$, goat anti-rabbit $800 \mathrm{cw}$, donkey anti-goat $800 \mathrm{cw}$ (see Materials List). Lane labels: $L$ — ladder, $1 / 2$ — sample 1/2. Please click here to view a larger version of this figure. 
1. Place membrane(s) in a square Petri dish that contain approximately $30 \mathrm{ml}$ of Revitablot stripping buffer and are incubated between 5-20 min at room temperature with constant agitation.

2. Wash the membrane(s) 3 times in PBS then rescan on the imager to ensure complete removal of fluorescence has occurred - this step should be repeated after every strip.

3. Incubate membrane(s) for longer periods if fluorescence remains. Rescan the membrane after each strip to confirm that there is no secondary signal remaining.

NOTE: Membrane(s) should only be stripped and reprobed a maximum of 3 times in order to guarantee that the integrity of the membrane has not been compromised resulting in undesirable high background to signal ratio.

\section{Representative Results}

As QFWB sensitivity and the linear range of detection is greater than conventional ECL detection, there are a number of control measures that are crucial to ensure that accurate data is collected, thereby aiding effective interpretation. Firstly, the inclusion of positive control samples as shown in Figure 1. Secondly, optimization of transfer to guarantee equivalent movement of high and low molecular weight proteins from the gel to the membrane as exhibited in Figure 2. Thirdly, optimization of antibodies, especially secondary antibodies whose optimization is often overlooked, but which can produce non-specific banding capable of interfering with correct interpretation of protein(s) of interest. See Figure 3. Fourthly, it may also be the case that when a protein appears undetectable but is expected to be present, this may also be a secondary antibody issue which can be corrected by simply using a secondary raised in a different species host. See Figure 4. Fifthly, total protein labeling and analysis is a far more robust and quantifiable method in comparison to the use of traditional single protein(s) that are ubiquitously expressed for internal reference standards ${ }^{3}$. Many of these single proteins have been found to be differentially expressed in models of neurodegenerative diseases as well as between different tissue samples and the uniformity of expression can alter within the same tissue ${ }^{3}$. Therefore, production of a loading control gel will confirm the uniformity of sample load when combined with a total protein analysis by comparing and quantifying the protein load in each lane at various molecular weights ranges measured against each sample to indicate standard error as demonstrated in Figure 6. Importantly, all of these troubleshooting techniques and controls are only as effective as the sensitivity and consistency of the analysis tools applied by the operator (Figure 5). Finally this technique lends itself to stripping and re-probing of membranes with more flexibility than ECL due to factors including but not limited to increased sensitivity, reduced background, dual color detection and membrane stability under long term storage conditions. See Figure 7.

\section{Discussion}

Due consideration and planning is essential prior to any experiment and can ultimately determine the success of the technique used. The advancements in protein detection using WB can present a plethora of potential stumbling blocks when trying to choose the appropriate antibodies, transfer and visualization methods to use. Fortunately, using a careful checklist and appropriate control measures QFWB can be used routinely to determine protein presence and increasingly subtle expression differences between samples. This protocol provides a comprehensive guide to fluorescent quantitative western blotting as well as a few troubleshooting strategies to avoid and/or overcome some of the many common pitfalls associated with it.

The critical steps employed to maintain sensitivity and obtain truly quantifiable and comparable measurements include: 1) robust protein extraction from tissue samples; 2) sample preparation; 3) accurate protein loading determined by total protein analysis; 4) optimal transfer of proteins using I-Blot; 5) preparation of primary and secondary antibodies in blocking buffer containing $0.1 \%$ Tween20, and 6 ) correct visualization and analysis using an infrared imager and associated software.

Infrared fluorescent detection is truly quantitative and provides greater sensitivity compared with more traditional ECL detection techniques ${ }^{3,11}$. This detection system is multi faceted, and as such is not limited to QFWB. This system is capable of imaging of immunohistological labeling at low power allowing visualization and quantification of whole tissue sections ${ }^{12}$. This is one area of potential future development in terms of resolution which could see far red imaging rivaling conventional immunofluorescence capturing with conventional microscopes in terms of quantitative assessment.

However, with greater sensitivity to subtle changes in protein expression it is crucial to ensure variability is kept to a minimum and control measures are stringent with robust protocols. This begins with rigorous protein extraction from the tissue sample followed by production of total protein stained gels to provide assurance that sample loading is uniform, optimization of primary and secondary antibodies in order to determine if detection is real and testing the manufacturer's guidelines with regard to transfer times to obtain efficient protein transfer.

Nevertheless, even when conditions for WB are optimized, there may still problems associated with running westerns that may not have been fully explored here. These include but are not limited to factors including protein solubilization and choice of extraction buffer. Some buffers can interfere with protein concentration assays, and some tissues are particularly difficult to solubilize, requiring more robust techniques such as the use of automated macerating sealed containers such as $\mathrm{M}$ tubes together with a Macs dissociator. In addition, simple control measures for storage of extracted and non-extracted material at $-80^{\circ} \mathrm{C}$ can be the difference between obtaining optimal labeling immediately after extraction and having poor results weeks later.

Modern QFWB methods are proven to be more sensitive for capturing subtle differences in protein expression and are more versatile allowing simultaneous dual labeling ${ }^{3}$ when compared to older techniques such as ECL. It is vital that western blotting protocols are robust and readily repeatable for accurate quantification and statistical analysis. This protocol is sensitive and robust enough to be used routinely for detection of proteins across a variety of different tissue samples and species ${ }^{3}$ and allows quantification of low and high abundance proteins within the same QFWB therefore reducing consumable usage as well as time per experiment ${ }^{17}$. In addition, the increased sensitivity of this technique allows validation of increasingly popular-omic studies ${ }^{9,14}$ however accuracy is crucial and inclusion of appropriate control measures must be adhered to thereby avoiding erroneous data acquisition. 


\section{Disclosures}

The authors have nothing to disclose.

\section{Acknowledgements}

We would like to thanks the following for financial support: BBSRC Institute Strategic Programme Funding - CF \& TMW; BBSRC East Bio DTP funding - LG; The Darwin Trust of Edinburgh - MLH. We would also like to thank Dr Barry McColl for permission to include the TREM2 optimization in this manuscript.

\section{References}

1. Towbin, H., Staehelin, T., Gordon, J. Electrophoretic transfer of proteins from polyacrylamide gels to nitrocellulose sheets: procedure and some applications. Biotechnology. 1992 (24), 145-9 (1979).

2. Walker, J.M. Protein Protocols on CD-ROM. Humana Press Inc., Totowa, NJ, USA (1998).

3. Gallagher, S., Winston, S.E., Fuller, S.A., Hurrell, J.G. Immunoblotting and immunodetection. Curr Protoc Mol Biol. Chapter 10, Unit 10.8. (2008).

4. Eaton, S.L. et al. Total protein analysis as a reliable loading control for quantitative fluorescent Western blotting. PLoS One. 8 (8), e72457 (2013).

5. Suzuki, O., Koura, M., Noguchi, Y., Uchio-Yamada, K., Matsuda, J. Use of sample mixtures for standard curve creation in quantitative Western blots. Exp. Anim. 60, 193-196 (2011).

6. Colella, A.D., et al. Comparison of Stain-Free gels with traditional immunoblot loading control methodology. Anal Biochem. 430 (2), $108-10$ (2012).

7. Zellner, M., et al. Fluorescence-based Western blotting for quantitation of protein biomarkers in clinical samples. Electrophoresis. 29, 36213627 (2008).

8. Kielar, C., et al. Molecular correlates of axonal and synaptic pathology in mouse models of Batten disease. Hum Mol Genet. 18, 4066-4080 (2009).

9. Mutsaers, C.A., Lamont, D.J., Hunter, G., Wishart, T.M., Gillingwater, T.H. Label-free proteomics identifies Calreticulin and GRP75/Mortalin as peripherally accessible protein biomarkers for spinal muscular atrophy. Genome Med. 5 (10), 95 (2013).

10. Wishart, T.M., et al. Dysregulation of ubiquitin homeostasis and $\beta$-catenin signaling promote spinal muscular atrophy. J Clin Invest. 124 (4), 1821-34, doi: 10.1172/JCI71318.(2014).

11. Mathews, S.T., Plaisance, E.P., Kim, T. Imaging systems for westerns: chemiluminescence vs. infrared detection. Methods Mol Biol. 536, 499-513. doi: 10.1007/978-1-59745-542-8_51 (2009).

12. Hawes, J.J., Brunzell, D.H., Wynick, D., Zachariou, V, Picciotto, M. R. GalR1, but not GalR2 or GalR3, levels are regulated by galanin signalling in the locus coeruleus through a cyclic AMP-dependent mechanism. J Neurochem. 93 (5), 1168-1176 (2006).

13. Bond, D., Primrose, D.A., Foley, E. Quantitative evaluation of signaling events in Drosophila S2 cells. Biol Proceed Online. 10 (1), $20-28$ (2008).

14. Wishart, T.M., et al. Differential proteomics analysis of synaptic proteins identifies potential cellular targets and protein mediators of synaptic neuroprotection conferred by the slow Wallerian degeneration (WIds) gene. Mol Cell Proteomics. 6, 1318-1330 (2007).

15. Silva, J.M., McMahon, M. The fastest western in town: a contemporary twist on the classic western blot analysis. J. Vis. Exp. 84, e51149, doi:10.3791/51149 (2014). 